\title{
Tight Glycemic Control in Intensive Care: From engineering to clinical practice change
}

\author{
J.G. Chase ${ }^{1}$, A.J. Le Compte ${ }^{1}$, A. Evans ${ }^{1}$, L. Ward ${ }^{1}$, J. Steel ${ }^{1}$, C.S. Tan $^{1}$, C.G. Pretty ${ }^{1}$, S. Penning ${ }^{2}$, T. \\ Desaive $^{2}$, G.M. Shaw ${ }^{3}$ \\ ${ }^{1}$ Univ of Canterbury, Dept of Mechanical Eng, Centre for Bio-Engineering, Christchurch, New Zealand \\ ${ }^{2}$ University of Liege, Cardiovascular Research Centre, Liege, Belgium \\ ${ }^{3}$ Dept of Intensive Care, Christchurch Hospital, Christchurch, New Zealand
}

\begin{abstract}
Tight glycemic control (TGC) is prevalent in critical care. Providing safe, effective TGC has proven very difficult to achieve with clinically derived protocols. The problem is exacerbated by extreme patient variability and the need to minimize clinical effort and burden. These ingredients make an ideal scenario for model-based methods to provide optimised solutions. This paper presents the development, clinically validated virtual trials optimisation, and initial clinical implementation of a stochastic targeted (STAR) TGC method and framework. It is compared to a prior successful, modelderived, less flexible and dynamic TGC protocol (SPRINT). The use of stochastic models to safely forecast a range of glucose outcomes over 1-3 hours ensures better performance, more dynamic use of the range of insulin and nutrition inputs and thus better glycemic performance and safety from hypoglycemia, the latter of which was reduced by 3.0x times. Hence, the paper presents an overall engineering approach to TGC from engineering models to clinical implementation and ongoing clinical practice change.
\end{abstract}

Keywords- ICU, TGC, glycemic control, control systems, modelling, hypoglycemia, clinical trials.

\section{INTRODUCTION}

Hyperglycaemia is prevalent in critical care, associated with increased cost, morbidity and mortality [1], due to several factors relating to the stress of patient condition [2]. Effective tight glycaemic control (TGC) can significantly reduce negative outcomes by modulating nutrition and/or insulin administration [3, 4]. However, consistent, effective TGC remains elusive [5].

A range of prior work successfully developed and validated metabolic system models [6, 7] and implemented a 'model-derived' protocol, SPRINT [3]. SPRINT is currently employed in the Christchurch ICU and effectively reduced mortality by $20-45 \%$ and per patient costs by $\$ 1000-2000$ per-patient [3]. It has also been shown to reduce the rate and severity of organ failure by $10 \%$ [8]. Unlike all other published protocols with similar glycaemic targets [5], it also reduced the incidence of hypoglycaemia by $50 \%$.
However, SPRINT has a fixed glycaemic target and lacks flexibility in several areas. It also offers no forecasting capabilities, is not easily customised to individual patients, and thus lacks the capacity to account for the wide range of metabolic variability observed in ICU patients $[9,10]$

This paper presents a novel, model-based, software control algorithm for the implementation of patient-specific TGC. It achieves accuracy of control similar to, or exceeding, that delivered by SPRINT, while reducing measurements and clinical effort. Uniquely, it can guarantee a reduced, clinically specified risk of hypoglycaemia. These outcomes are delivered within a flexible framework offering a range of clinical approaches and targets

\section{Methods}

\section{A. Patients}

Virtual trials are a safe means of optimising glycaemic control robustness, performance, safety from hypoglycaemia, and clinical burden [7, 11]. Done in silico, it is also much faster and lower cost than a strictly clinical protocol design approach. The TGC algorithm presented is tested using clinically validated [7] virtual patient simulations. The entire 371 patient SPRINT Cohort was used to create virtual patients for protocol design and analysis.

\section{B. Model}

The metabolic system model used is defined [7]:

$$
\begin{aligned}
& G=-p_{Q} G-S_{l} G \frac{Q}{1+v_{Q} Q}+\frac{p\left(t^{t}\right)+E G P-C N s}{v_{E}} \\
& \phi=-k Q+k I \\
& I=-\frac{\mathrm{m} I}{1+a_{l} l}+\frac{u_{2 x}(t)}{v_{2}}+3 e^{-\left(u_{\mathrm{gx}}(\mathrm{d}) \omega V_{2}\right)} \\
& P(t)=\min \left(d_{2} P_{2}, P_{\max }\right) \\
& p_{2}=-\min \left(d_{2} P_{2}, P_{\max }\right)+d_{1} P_{1} \\
& P_{1}=-d_{1} P_{1}+D(t)
\end{aligned}
$$

Model parameters and values are described in Table 1. Insulin sensitivity $S_{I}$ is identified hourly enabling virtual patients and trials $[6,7]$. 
Table 1: Model parameters and values

\begin{tabular}{|c|c|c|}
\hline & Description & Values \\
\hline$G$ & Blood glucose level & $(\mathrm{mmol} / \mathrm{L})$ \\
\hline$p_{G}$ & $\begin{array}{l}\text { Insulin independent glucose removal } \\
\text { (excluding central nervous system } \\
\text { uptake) and the suppression of } E G P \\
\text { from } E G P_{b} \text { with respect to } G\end{array}$ & $0.006\left(\mathrm{~min}^{-1}\right)$ \\
\hline$\alpha_{G}$ & $\begin{array}{l}\text { Saturation parameter for insulin medi- } \\
\text { ated glucose removal }\end{array}$ & $1 / 65(\mathrm{~L} / \mathrm{mU})$ \\
\hline$S_{I}$ & $\begin{array}{l}\text { Insulin mediated glucose removal and } \\
\text { the suppression of } E G P \text { from } E G P_{b} \\
\text { with respect to } G \text { and } Q\end{array}$ & $(\mathrm{~L} / \mathrm{mU} / \mathrm{min})$ \\
\hline$Q$ & Interstitial insulin concentration & $(\mathrm{mU} / \mathrm{L})$ \\
\hline$P(t)$ & $\begin{array}{l}\text { Glucose appearance in plasma from } \\
\text { dextrose intake }\end{array}$ & $(\mathrm{mmol} / \mathrm{min})$ \\
\hline$E G P$ & Endogenous glucose production & $\begin{array}{c}1.16 \\
(\mathrm{mmol} / \mathrm{min})\end{array}$ \\
\hline$C N S$ & Central nervous system glucose uptake & $\begin{array}{c}0.3 \\
(\mathrm{mmol} / \mathrm{min})\end{array}$ \\
\hline$V_{G}$ & Plasma glucose distribution volume & $13.3(\mathrm{~L})$ \\
\hline$k$ & Interstitial insulin transport rate & $\begin{array}{c}-\ln (0.5) / 35 \\
\left(\min ^{-1}\right) \\
\end{array}$ \\
\hline$I$ & Plasma insulin concentration & $(\mathrm{mU} / \mathrm{L})$ \\
\hline$n$ & Plasma insulin decay rate & $\begin{array}{l}0.16\left(\min ^{-1}\right) \\
\left(\min ^{-1}\right)\end{array}$ \\
\hline$\alpha_{I}$ & $\begin{array}{l}\text { Saturation parameter for plasma } \\
\text { insulin clearance }\end{array}$ & $\begin{array}{c}1.7 \times 10^{-3} \\
(\mathrm{~L} / \mathrm{mU})\end{array}$ \\
\hline$u_{e x}(t)$ & Exogenous insulin & $(\mathrm{mU} / \mathrm{min})$ \\
\hline$V_{I}$ & Plasma insulin distribution volume & $3.15(\mathrm{~L})$ \\
\hline$d_{2}$ & Glucose absorption rate from gut & $\begin{array}{c}-\ln (0.5) / 100 \\
\left(\min ^{-1}\right)\end{array}$ \\
\hline$P 2$ & Glucose level in gut & $(\mathrm{mmol})$ \\
\hline$P_{\max }$ & $\begin{array}{l}\text { Maximal glucose flux from gut to } \\
\text { plasma }\end{array}$ & $\begin{array}{c}6.11 \\
(\mathrm{mmol} / \mathrm{min}) \\
\end{array}$ \\
\hline P1 & Glucose level in stomach & $(\mathrm{mmol})$ \\
\hline$d_{1}$ & Glucose absorption rate from stomach & $\begin{array}{c}-\ln (0.5) / 20 \\
\left(\mathrm{~min}^{-1}\right)\end{array}$ \\
\hline$D(t)$ & Dextrose intake & $(\mathrm{mmol} / \mathrm{min})$ \\
\hline
\end{tabular}

Time-variable insulin sensitivity profiles $\left(\mathrm{S}_{\mathrm{I}}(\mathrm{t})\right)$, created from patient data, are identified hourly from clinical data [6]. $S_{I}$ has been extensively validated for in silico virtual patients and accurate predictions [7].

\section{Stochastic Model and Target-to-Range}

The Stochastic Targeted (STAR) TGC protocol presented optimises treatment based on a stochastic model $[9,10]$ of the potential variability in $S_{\mathrm{I}}(\mathrm{t})$ over the following 1-3 hours. This variability results in a range of likely BG outcomes for any given insulin and nutrition intervention, including the median, IQR and $90 \%$ confidence interval.

STAR modulates both insulin and nutrition, or just insulin for a fixed nutritional input. The controller examines all possible combinations for each of 1, 2 and 3 hour forward intervals, within certain constraints. Specifically:

- Insulin rates can increase by no more than $3 \mathrm{U} /$ hour
- Feed rate changes are limited to $\pm 20 \%$ of goal feed

Within these limits all possible insulin and nutrition interventions are considered to reach target.

The controller targets a clinically specified range. In this case, the range of 4.0-6.5 mol/L. For 1-hour interventions the $95^{\text {th }}$ percentile outcome is set on $6.5 \mathrm{mmol} / \mathrm{L}$. For 2 and 3 hour interventions, the $5^{\text {th }}$ percentile outcome is targeted to $4.5 \mathrm{mmol} / \mathrm{L}$ and recognises the potential loss of some control to a safer (than hypoglycemia) level above 6.5 $\mathrm{mmol} / \mathrm{L}$ in some cases.

In all cases, the $5^{\text {th }}$ percentile is never set lower than 4.0 $\mathrm{mmol} / \mathrm{L}$ guaranteeing a maximum risk of $5 \%$ of very moderate hypoglycemia less than this value.

\section{Analyses and Clinical Proof of Concept}

The following virtual trials were run to assess STAR:

- Measurement interval limited to 1 hour - Best Case

- Measurement interval limited to 2 hours (when available) - Intermediate Case

- Measurement interval not limited (up to 3 hours) and longest available selected - Minimum Clinical Effort Case

The 2 hour limited intervals case is the closest comparator to the clinical SPRINT data.

Clinical pilot trials were run for 7 patients. Their data is presented similarly for broad comparison. Each patient was individually consented, with approval granted by the NZ South Island Regional Ethics Committee.

\section{RESUlTS AND DisCUSSION}

\section{A. Virtual Trial Results}

Tables 2-3 show the performance and safety from hypoglycemia results for the in silico virtual trials. It is clear that performance is comparable or improved from SPRINT, as desired. More importantly, safety from hypoglycemia is significantly reduced at all measurement intervals compared to SPRINT, particularly in numbers of patients exposed to severe hypoglycemia $(\mathrm{BG}<2.2 \mathrm{mmol} / \mathrm{L})$, which is as expected given the stochastic targeting methods used.

Table 2: Performance results in silico 1, 2, 3 hour intervals

\begin{tabular}{|l|c|c|c|c|}
\hline & 1 hour & 2 hour & 3 hour & SPRINT \\
\hline $\begin{array}{l}\text { BG median [IQR] } \\
\text { (mmol/L) }\end{array}$ & $\begin{array}{c}5.8 \\
{[5.3-6.4]}\end{array}$ & $\begin{array}{c}5.8 \\
{[5.2-6.8]}\end{array}$ & $\begin{array}{c}6.1 \\
{[5.3-7.1]}\end{array}$ & $\begin{array}{c}5.7 \\
{[5.0-6.6]}\end{array}$ \\
\hline \% BG: $4.0-6.5$ & 75 & 66 & 58 & 70 \\
\hline \% BG: $5.0-6.5$ & 64 & 50 & 45 & 50 \\
\hline \% BG: $4.0-8.0$ & 93 & 87 & 84 & 88 \\
\hline
\end{tabular}


Table 3: Safety from hypoglycemia performance (Total is from 371)

\begin{tabular}{|l|l|l|l|l|}
\hline & 1-hour & 2-hour & 3 -hour & SPRINT \\
\hline Total \#<2.2mmol/L & 6 & 6 & 8 & 13 \\
\hline$\%$ BG $<4.0 \mathrm{mmol} / \mathrm{L}$ & 1.1 & 3.0 & 3.5 & 3.8 \\
\hline Total \#<4.0mmol/L & 129 & 187 & 179 & 283 \\
\hline
\end{tabular}

For clarity the patient-specific cumulative distribution functions (CDFs) are shown for SPRINT and STAR 2 hour measurement intervals in Figures 1-4. These figures show the CDF for each patient as grouped around the median patient. The $5^{\text {th }}$ and $95^{\text {th }}$ percentile patients represent the outliers of over and under control of BG, while the $25^{\text {th }}$ and $75^{\text {th }}$ percentiles show the band within which the central tendency and overall expected outcome lies. The 2-hour measurement interval analysis of STAR, in which the longest allowed measurement interval is 2-hours comparable to SPPRINT, shows, as in Table 2, very similar results. As expected, using 3-hour intervals results in a slight loss of control and using only 1-hour intervals provides very tight control. The tradeoff is in clinical effort, where 2-3 hour intervals are desired in most situations to reduce burden on nursing staff.

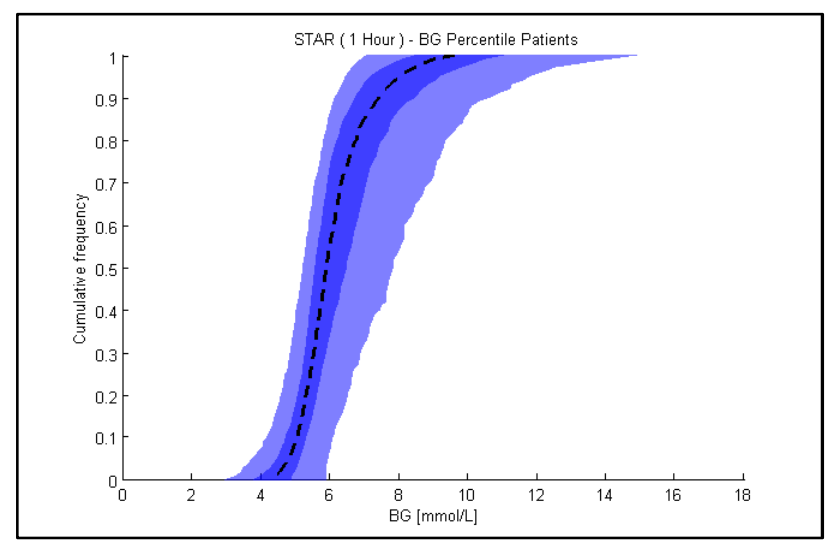

Figure 1: STAR (1 hour) patient specific BG CDFs

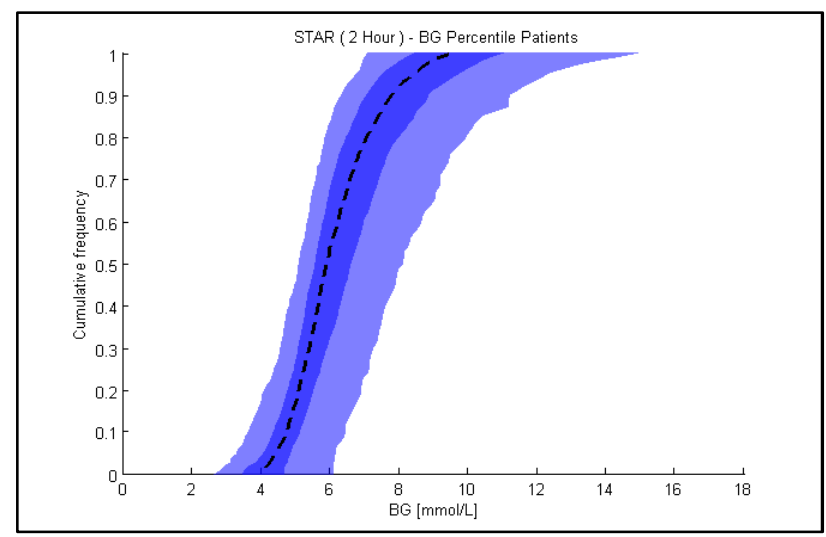

Figure 2: STAR (2 hour) patient specific BG CDFs

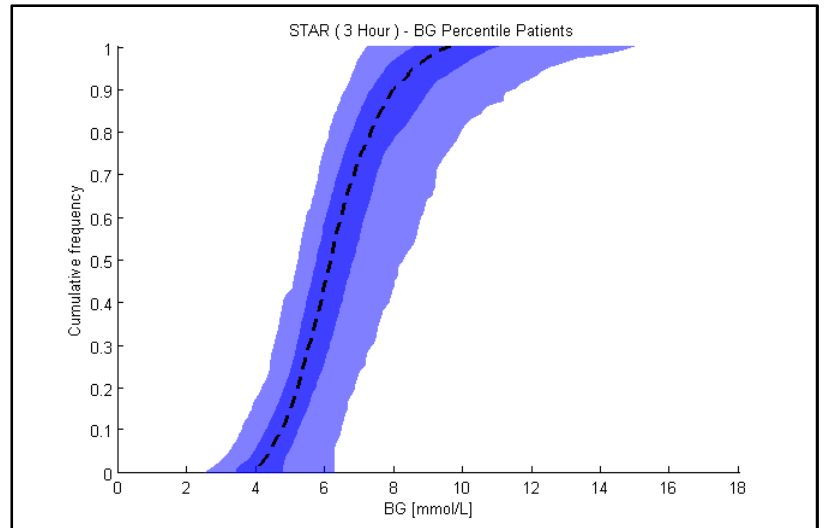

Figure 3: STAR (3 hour) patient specific BG CDFs

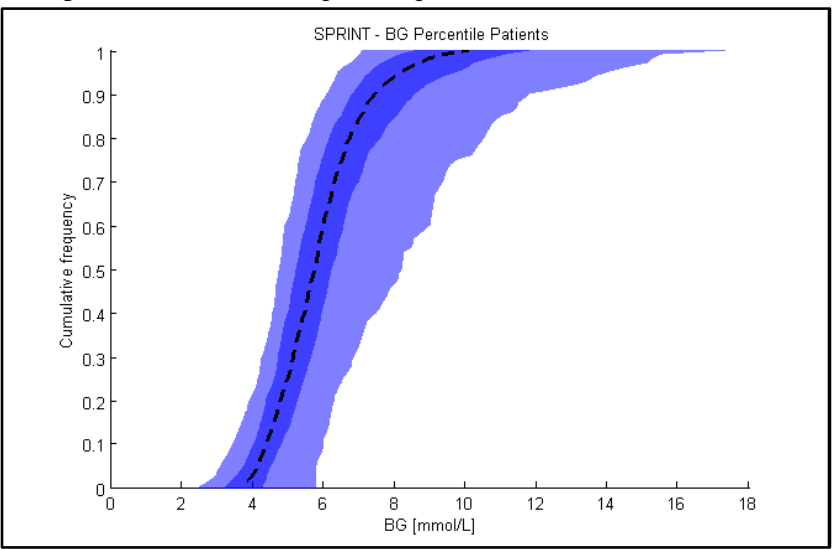

Figure 4: SPRINT clinical, patient specific BG CDFs

In particular, the reduced risk of hypoglycaemia across patients can also be seen. Figures 1-4 compare the median patient across each case. The $5^{\text {th }}$ percentile patients show safety. It is clear that the median SPRINT patient had some $\mathrm{BG}<4.0 \mathrm{mmol} / \mathrm{L}$, where the median STAR patient did not, indicating greater safety with this approach for greater numbers of patients, as well as comparable or better overall performance.

\section{B. Clinical Proof of Concept Trials}

At the time of this writing, 7 patients had been consented and been treated with STAR for the entire length of ICU stay requiring glycemic control. Tables 4-5 summarise the results from these trials in the same format as Tables 2-3 for comparison. For comparison, the results from virtual trials on these 7 patients, using SPRINT with the nutrition given in these clinical trials, and as would have been given if SPRINT were used normally, are shown. 
Table 4: Performance results STAR trials versus SPRINT in silico

\begin{tabular}{|l|c|c|c|}
\hline & STAR & $\begin{array}{c}\text { SPRINT w/ feed from } \\
\text { STAR }\end{array}$ & SPRINT \\
\hline $\begin{array}{l}\text { BG median } \\
{[\mathrm{IQR}]}\end{array}$ & $\begin{array}{c}5.9 \\
{[5.2-68]}\end{array}$ & $\begin{array}{c}5.7 \\
{[4.7-6.8]}\end{array}$ & $\begin{array}{c}5.3 \\
{[4.6-6.0]}\end{array}$ \\
\hline \% BG: $4.0-6.5$ & 63 & 57 & 72 \\
\hline \% BG: $5.0-6.5$ & 76 & 68 & 79 \\
\hline \% BG: $4.0-8.0$ & 90 & 84 & 87 \\
\hline
\end{tabular}

Table 5: Safety of STAR versus SPRINT in silico

\begin{tabular}{|l|c|c|c|}
\hline & STAR & $\begin{array}{c}\text { SPRINT w/ feed from } \\
\text { STAR }\end{array}$ & SPRINT \\
\hline No. $<2.2 \mathrm{mmol} / \mathrm{L}$ & 0 & 2 & 3 \\
\hline$\% \mathrm{BG}<4.0 \mathrm{mmol} / \mathrm{L}$ & 4.5 & 10.4 & 12.5 \\
\hline
\end{tabular}

With respect to performance it is clear that STAR has comparably tight control, and exceeds SPRINT in the 4.0$8.0 \mathrm{mmol} / \mathrm{L}$ band. The difference is seen primarily in safety where there is no severe hypoglycemia in STAR, which had a minimum measurement of $3.8 \mathrm{mmol} / \mathrm{L}$ over all $404 \mathrm{BG}$ measurements. Equally, the percentage of measurements below $4.0 \mathrm{mmol} / \mathrm{L}$ is less than $5 \%$, as guaranteed by STAR, and 2.5-3.0 times less than for SPRINT.

These 7 patients covered 660 hours. There were 404 measurements, for an average of 14.5 measurements per day. This is reduced from 17/day in SPRINT and indicates a clinical time savings of $\sim 12-15 \mathrm{~min} /$ day, which is significant added up across shifts. It is also near the in silico prediction of 14/day using STAR 2-hour. Importantly, nursing staff tended not to choose 3-hourly measurements in these trials, for a range of reasons from safety to habit from SPRINT. Thus, 2-3 hour forecast intervals are highly acceptable clinically for both SPRINT and STAR, and equally effective in both cases versus longer intervals used elsewhere [11].

The SPRINT analyses in Tables 4-5 are somewhat different. The first uses the same nutrition rates as STAR used in its trials, so it thus compares the insulin administration choices only. It is far more variable and distinctly underperforms. The second analysis, uses SPRINT is comparable to its overall reported clinical performance and to STAR, excepting the relatively high rate of moderate hypoglycemia. Hence, STAR was less variable, as seen in Figures 1-4 as well with the steeper and more tightly grouped CDFs.

Regarding the two SPRINT comparison virtual trials, the insulin and feed administration was very different. In particular, SPRINT uses lower nutritional dextrose administration rates, which was seen in these virtual trials where the cohort median [IQR] dextrose administration was 4.5 [4.4, 4.9] g/hour for SPRINT and a much higher and more variable $6.8[5.8,8.7] \mathrm{g} /$ hour for STAR. These inputs were offset by insulin rates of $3.0[2.0,3.0] \mathrm{U} /$ hour for SPRINT and, an again much more variable, $2.5[0.0,6.0] \mathrm{U} /$ hour for STAR.

Hence, it is clear that the STAR controller achieves its better results by a more dynamic use of the full range of control inputs (dextrose and insulin administration). This ability is provided by the stochastic forecasting methods unique to this approach that enable more significant inputs, when warranted and provably safe via stochastic forecasting. As a result, the clinician receives comparable performance and better safety.

\section{Conclusions}

This research presented an overall stochastic targeted (STAR) approach to TGC in critical care. It shows how to take a method from a model and data, to analysis and into clinical practice as was done for SPRINT and now for STAR, by using clinically validated in silico virtual trials to safely optimise a protocol without clinical risk.

The overall STAR approach is unique in its stochastic forecasting ability. As a result, it can utilise more dynamic ranges of control inputs, more safely, and with less clinical intervention or burden. These are unique, compromise busting outcomes for the field. Hence, these results also validate the overall model-based approach presented that can rapidly take a clinical intervention from engineering and ideas to clinical practice change.

\section{REFERENCES}

1. Van den Berghe, G., et al., Analysis of healthcare resource utilization with intensive insulin therapy in critically ill patients. Crit Care Med, 2006. 34(3): p. 612-6.

2. McCowen, K.C., A. Malhotra, and B.R. Bistrian, Stress-induced hyperglycemia. Crit Care Clin, 2001. 17(1): p. 107-124.

3. Chase, J.G., et al., Implementation and evaluation of the SPRINT protocol for tight glycaemic control in critically ill patients: a clinical practice change. Crit Care, 2008. 12(2): p. R49.

4. Van den Berghe, G., et al., Intensive insulin therapy in the critically ill patients. N Engl J Med, 2001. 345(19): p. 1359-1367.

5. Griesdale, D.E., et al., Intensive insulin therapy and mortality among critically ill patients: a meta-analysis including NICE-SUGAR study data. Cmaj, 2009.

6. Hann, C.E., et al., Integral-based parameter identification for longterm dynamic verification of a glucose-insulin system model. Comput Methods Programs Biomed, 2005. 77(3): p. 259-270.

7. Chase, J.G., et al., Validation of a model-based virtual trials method for tight glycemic control in intensive care. Biomed Eng Online, 2010. 9: p. 84.

8. Chase, J.G., et al., Organ failure and tight glycemic control in the SPRINT study. Crit Care, 2010. 14(4): p. R154.

9. Lin, J., Lee, DS, Chase, JG, Hann, CE, Lotz, T and Wong, XW, Stochastic Modelling of Insulin Sensitivity Variability in Critical Care. Biomedical Signal Processing \& Control, 2006. 1: p. 229-242.

10. Lin, J., et al., Stochastic modelling of insulin sensitivity and adaptive glycemic control for critical care. Comput Methods Programs Biomed, 2008. 89(2): p. 141-52.

11. Lonergan, T., et al., A Simple Insulin-Nutrition Protocol for Tight Glycemic Control in Critical Illness: Development and Protocol Comparison. Diabetes Technol Ther, 2006. 8(2): p. 191-206. 